\title{
The condition of microscopic reversibility in Gibbs ensemble Monte Carlo simulations of phase equilibria
}

\author{
By LUIS F. RULL, GEORGE JACKSON $\dagger$ \\ Departamento de Física Atómica Molecular y Nuclear, Universidad de Sevilla, \\ Aptdo. 1065, Sevilla 41080, Spain \\ and BEREND SMIT \\ Koninklijke/Shell-Laboratorium Amsterdam, Postbus 38000, \\ 1030 BN Amsterdam, The Netherlands
}

(Received 22 December 1994; accepted 16 January 1995)

\begin{abstract}
The condition of microscopic reversibility, also referred to as detailed balance, is examined in the context of Monte Carlo simulations in the Gibbs ensemble. The technique is used widely in the simulation of phase equilibria for liquids and their mixtures, and represents an invaluable tool in the area. The two coexisting phases are simulated as separate subsystems by performing three distinct Monte Carlo moves which include random displacements of particles in each subsystem, random changes in volume, and random transfers of particles between the two subsystems. Here, the particle transfer step of the Gibbs ensemble technique, as commonly implemented, is shown to be reversible. Other valid reversible criteria are presented for pure fluids and mixtures. The vapour-liquid equilibria of the pure square-well fluid with a range of $\lambda=1.5$ are examined with the various criteria. As expected, the choice of criteria makes little difference for pure fluids. The results are also presented of liquid-liquid immiscibility for a symmetrical square-well mixture with range $\lambda=1 \cdot 5$ in which the unlike interactions are purely repulsive. For this mixture the various reversible algorithms for particle transfers give essentially equivalent results, although the efficiency in sampling phase space is sometimes quite different.
\end{abstract}

\section{Introduction}

A lot of effort has been employed in the development of techniques for the simulation of phase equilibria of fluids and fluid mixtures [1]. Indirect methods invariably involve calculating the chemical potential or free energy of each phase during a series of Monte Carlo or molecular dynamics simulations. When two phases are found to have the same temperature, pressure and chemical potential they are in equilibrium. However, the overall procedure is tedious and time-consuming. Direct methods for simulating phase equilibria are generally quicker, more efficient, and easier to apply than the indirect methods. One method involves simulating the two coexisting phases separated by an interface in a single simulation box. Bulk phase equilibria can be represented for sufficiently large system sizes, but the confinement often influences the properties of the fluid, especially for temperatures close to the critical point. More recently, Panagiotopoulos [2] has developed the so-called Gibbs ensemble Monte Carlo technique. In this direct method, typical regions of the two coexisting homogeneous phases are simulated as separate subsystems, without the

$\dagger$ This work was undertaken while on study leave from the Department of Chemistry, University of Sheffield, Sheffield S3 7HF, UK; all correspondence to this address. 
presence of an interface. The simulation involves three Monte Carlo moves including random displacements of particles in each subsystem, random changes in volume, and random transfers of particles between the two subsystems. The Gibbs ensemble simulations are a good representation of bulk behaviour since, in this case, there is no interface and the confinement problem does not arise. The method also allows a closer approach to the critical point.

The Gibbs ensemble Monte Carlo technique has become the most commonly used and versatile method for the direct simulation of phase equilibria. It has already been used to study a wide variety of fluids and fluid mixtures, the results of which have been extensively reviewed $[1,3,4]$. The technique is just as easy to apply to vapour-liquid coexistence in pure fluids as to vapour-liquid coexistence in mixtures.

Quite recently, Gibbs ensemble simulations were undertaken in order to determine the phase equilibria of mixtures containing square-well particles [5]. The modified algorithm which was used led us to examine the condition of microscopic reversibility, also called detailed balance, for the particle transfer stage of the Gibbs ensemble iechnique. One must always ensure that this condition is satisfied when Monte Carlo algorithms are proposed in order to guarantee that a proper Markov chain of states is generated [6]. In the following section we show that the technique as originally devised complies with the condition of microscopic reversibility [6]. Alternative reversible criteria are also proposed. In the case of the vapour-liquid equilibria of a pure square-well fluid with range $\lambda=1.5$ we show that the various algorithms make little difference to the calculated phase equilibria for pure fluids.

Any possible differences could, however, be highlighted in the case of mixtures, where the numbers of particles of a given component in the coexisting phases are often very different. It turns out that the various algorithms proposed for mixtures all reproduce the phase equilibria obtained using the original algorithm of Panagiotopoulos et al. [7]. This will be demonstrated in section 3 for a symmetrical square-well mixture with range $\lambda=1.5$ in which the unlike interactions are purely repulsive, corresponding to hard-sphere interactions. The lack of unlike attractive interactions in such a system results in a substantial region of liquid-liquid immiscibility at high pressures (see [8]).

\section{Gibbs ensemble Moute Carlo simulations of pure fluids}

First we review the Gibbs ensemble Monte Carlo technique in order to set the scene for the rest of the discussion. The thorough statistical mechanical description of the technique presented by Smit and co-workers $[9,10]$ has been adopted.

In the Gibbs ensemble of a pure fluid the $N$ particles are at a constant temperature $T$ and in a volume $V$, as for the canonical ensemble. However, the system is divided into two separate subsystems $a$ and $b$ with volumes $V^{a}$ and $V^{b}$ and numbers of particles $N^{a}$ and $N^{b}$ so that $V=V^{a}+V^{b}$ and $N=N^{a}+N^{b}$. The subsystems are allowed to interchange particles and the volumes allowed to fluctuate in order to satisfy the conditions of phase equilibrium, i.e., that the temperature, pressure and chemical potential of the subsystems are equal.

The partition function for the Gibbs ensemble is given by

$$
\begin{aligned}
Q_{N V T}^{\mathrm{Gibbs}}= & \frac{1}{N ! \Lambda^{3 N} V_{0}} \sum_{N^{a}=0}^{N} \frac{N !}{N^{a} ! N^{b} !} \int_{0}^{V} \mathrm{~d} V^{a} \\
& \times \int_{V^{a}} \mathrm{~d}\left(\mathbf{r}^{a}\right)^{N^{a}} \exp \left(-\frac{U^{a}\left(N^{a}\right)}{k T}\right) \int_{V^{b}} \mathrm{~d}\left(\mathbf{r}^{b}\right)^{N^{b}} \exp \left(-\frac{U^{b}\left(N^{b}\right)}{k T}\right),
\end{aligned}
$$


where $A$ is the thermal de Broglie wavelength, $V_{0}$ is a basic unit of volume chosen to render the partition function dimensionless [11], $k$ is the Boltzmann constant, $\left(\mathbf{r}^{j}\right)^{N^{j}}$ represents the positions of particles in subsystem $j$, and $U^{j}\left(N^{j}\right)$ is the energy of subsystem $j$. After introducing the re-scaled coordinates $\xi^{j}==\mathbf{r}^{j} / L^{j}$, where $L^{j}$ is the box length of the simulated subsystem $j$, the partition function of the total systems can be written as

$$
\begin{aligned}
Q_{N Y T}^{\text {Gibbs }}= & \frac{1}{N ! \Lambda^{3 N} V_{0}} \sum_{N^{a}=0}^{N} \frac{N !}{N^{a} ! N^{b} !} \int_{0}^{V} \mathrm{~d} V^{a}\left(V^{a}\right)^{N^{a}}\left(V^{b}\right)^{N^{b}} \\
& \times \int_{V^{a}} \mathrm{~d}\left(\xi^{a}\right)^{N^{a}} \exp \left(-\frac{U^{a}\left(N^{a}\right)}{k T}\right) \int_{V^{b}} \mathrm{~d}\left(\xi^{b}\right)^{N^{b}} \exp \left(-\frac{U^{b}\left(N^{b}\right)}{k T}\right) .
\end{aligned}
$$

Hence, the average of a function $f$ in the Gibbs ensemble is given by

$$
\begin{aligned}
\langle f\rangle_{N V T}^{\text {Gibbs }}= & \frac{1}{Q_{N V T}^{\text {Gibbs }}} \frac{1}{N ! \Lambda^{3 N} V_{0}} \sum_{N^{a}=0}^{N} \frac{N !}{N^{a} ! N^{b} !} \int_{0}^{V} \mathrm{~d} V^{a}\left(V^{a}\right)^{N^{a}}\left(V^{b}\right)^{N^{b}} \\
& \times \int_{V^{a}} \mathrm{~d}\left(\xi^{a}\right)^{N^{a}} \exp \left(-\frac{U^{a}\left(N^{a}\right)}{k T}\right) \int_{V^{b}} \mathrm{~d}\left(\xi^{b}\right)^{N^{b}} \exp \left(-\frac{U^{b}\left(N^{b}\right)}{k T}\right) f
\end{aligned}
$$

It is clear from an inspection of equation (3) that the corresponding probability distribution for the ensemble is proportional to a pseudo-Boltzmann factor given by

$$
\mathscr{P}^{\mathrm{Gibbs}}=\exp \left[\ln \left(\frac{N !}{N^{a} ! N^{b} !}\right)+N^{a} \ln V^{a}+N^{b} \ln V^{b}-\frac{U^{a}\left(N^{a}\right)}{k T}-\frac{U^{b}\left(N^{b}\right)}{k T}\right] .
$$

The acceptance probability of the three types of move that are attempted in a Gibbs ensemble simulation are governed by expression (4). The first involves the random displacement of particles in each subsystem using the well established canonical NVT Metropolis scheme [12] so that new configurations of subsystem $j$ are generated with a probability given by $\min \left(1, \mathscr{P}^{\mathrm{d} j}\right)$ where

$$
\mathscr{P}^{\mathrm{d}^{j}}=\exp \left(-\frac{\Delta U^{j}}{k T}\right)
$$

Here, $\Delta U^{j}$ represents the change in energy in subsystem $j$ caused by the displacement. The second type of move involves a random change $\Delta V$ in the volume of subsystem $a$ and a corresponding change $-\Delta V$ in the volume of subsystem $b$ so that the new configurations are generated with a probability given by $\min \left(1, \mathscr{P}^{\mathrm{v}}\right)$ where

$$
\mathscr{P}^{\mathrm{V}}=\exp \left[N^{a} \ln \left(\frac{V^{a}+\Delta V}{V^{a}}\right)+N^{b} \ln \left(\frac{V^{b}-\Delta V}{V^{b}}\right)-\frac{\Delta U^{a}}{k T}-\frac{\Delta U^{b}}{k T}\right],
$$

which is identical to the prescription of Wood [13] for constant pressure NPT simulations. The third type of move involves a particle transfer between the two subsystems; for a transfer of a particle from subsystem $a$ to subsystem $b$, the configurations are generated with a probability given by $\min \left(1, \mathscr{P}^{t}\right)$ where

$$
\mathscr{P}^{\mathrm{t}}=\exp \left[\ln \left(\frac{N^{a} V^{b}}{\left(N^{b}+1\right) V^{a}}\right)-\frac{\Delta U^{a}}{k T}-\frac{\Delta U^{b}}{k T}\right]
$$


This particle transfer is similar to the creation and annihilation of particles in the grand canonical $\mu V T$ ensemble [14].

A cycle in a Gibbs ensemble Monte Carlo simulation usually involves $N^{a}$ and $N^{b}$ particle displacements in subsystems $a$ and $b$, one coupled volume change, and $\mathscr{O}(10 \times N)$ particle transfers between the two subsystems. Typically, $\mathscr{O}(100 \times N)$ cycles are performed. In order to ensure that the condition of microscopic reversibility is fulfilled, the three types of move have to be selected at random with fixed probabilities chosen so that the appropriate ratios of each type of move are obtained [4]. The particle-displacement and volume-change moves of Gibbs ensemble simulations can themselves be seen to satisfy the conditions of microscopic reversibility, since these moves are no different from those in standard simulations in the canonical and isothermal-isobaric ensembles [6]. This is not so obvious for the particle transfer move, as will become clear from the ensuing discussion.

With the original algorithm of Panagiotopoulos [2], which we refer to as algorithm 1, the donor and recipient subsystems for the transfer are first chosen at random, and then a random particle in the donor subsystem is transferred to a random position in the recipient subsystem. Let us consider the pure system in an overall configuration $\alpha$ with $N^{a}$ and $N^{b}$ particles in subsystems $a$ and $b$. A new overall configuration $\beta$ is generated by transferring a particle from subsystem $a$ to $b$ so that there are now $N^{a}-1$ and $N^{b}+1$ particles in subsystems $a$ and $b$. The probability of this transfer is proportional to

$$
\mathscr{P}_{\alpha \beta}=N^{a} ! N^{b} ! \times \mathscr{P}^{\text {subsystem } a} \times \mathscr{P}_{\alpha}^{\text {particle } a} \times \mathscr{P}^{\text {position } b} \times \mathscr{P}_{\alpha}^{\text {Gibbs }} \times \mathscr{P}_{\alpha \beta}^{\text {criterion }}
$$

where $\mathscr{P}^{\text {subsystem } a}$ is the probability of choosing subsystem $a$ as the donor for the transfer, $\mathscr{P}_{\alpha}^{\text {particle } a}$ is the probability that a particle is chosen from the $N^{a}$ of subsystem $a$ in configuration $\alpha, \mathscr{P}^{\text {position } b}$ is the probability of selecting a position for insertion in subsystem $b, \mathscr{P}_{\alpha}^{\text {Gibbs }}$ is the probability of the total system being in configuration $\alpha$, and $\mathscr{P}_{\alpha \beta}^{\text {criterion }}$ is the acceptance criterion used for the particle transfer. The probabilities of choosing either subsystem $a$ or $b$ as a donor or recipient for the transfer are usually equal so that $\mathscr{P}^{\text {subsystem } a}=\mathscr{P}^{\text {subsystem } b}=\frac{1}{2}$. Consequently, these factors will not be present in the final acceptance criterion for the transfer. The probability of choosing a particle in conventional $N V T$ and $N P T$ simulations is fixed, i.e., $\mathscr{P}^{\text {particle }}=1 / N$, and hence the factor just forms part of the constant of proportionality in the overall probability for the Monte Carlo move. However, in the case of a Gibbs ensemble simulation the number of particles in each subsystem changes, as does the probability of choosing a particular particle; in the case of our present example, the probability of choosing a particle from subsystem $a$ in configuration $\alpha$ is $\mathscr{P}_{\alpha}^{\text {particle } a}=1 / N^{a}$. Since the particles are inserted uniformly in each subsystem the probabilities of selecting a position for insertion in subsystems $a$ and $b$ are equal, i.e., $\mathscr{P}^{\text {position } a}=\mathscr{P}^{\text {position } b}$, which means that these terms will not appear in the overall acceptance criterion. The probability of finding the system in configuration $\alpha$ is obtained from equation (4). Finally, it should be noted that since the particles are indistinguishable, the labelling of the particles is not important. Since we will later impose the condition of microscopic reversibility we have to sum over all $N^{a} ! N^{b}$ ! possible permutations of the labels used in the actual simulation, and hence the multiplying factor in expression (8). This is essentially equivalent to using the partition function of a system of distinguishable particles (e.g., see [15]).

For the reverse move, i.e., the probability of transferring a particle from subsystem 
$b$ back to subsystem $a$,

$$
\mathscr{P}_{\beta \alpha}=\left(N^{a}-1\right) !\left(N^{b}+1\right) ! \times \mathscr{P}^{\text {subsystem } b} \times \mathscr{P}_{\beta}^{\text {particle } b} \times \mathscr{P}^{\text {position } a} \times \mathscr{P}_{\beta}^{\text {Gibbs }} \times \mathscr{P}_{\beta \alpha}^{\text {criterion }}
$$

where now $\mathscr{P}_{\beta}^{\text {particle } b}=1 /\left(N^{b}+1\right)$,

$$
\begin{aligned}
\mathscr{P}_{\beta}^{\text {Gibbs }}= & \exp \left[\ln \left(\frac{N !}{\left(N^{a}-1\right) !\left(N^{b}+1\right) !}\right)+\left(N^{a}-1\right) \ln V^{a}\right. \\
& \left.+\left(N^{b}+1\right) \ln V^{b}-\frac{U^{a}\left(N^{a}-1\right)}{k T}-\frac{U^{b}\left(N^{b}+1\right)}{k T}\right],
\end{aligned}
$$

$\mathscr{P}_{\beta \alpha}^{\text {criterion }}$ is the acceptance criterion used for the transfer, and the multiplying factor of $\left(N^{a}-1\right) !\left(N^{b}+1\right)$ ! takes into account the sum over all possible permutations of the labels for configuration $\beta$ with $N^{a}-1$ and $N^{b}+1$ particles in subsystems $a$ and $b$, respectively.

In order to satisfy the condition of microscopic reversibility, the probabilities of the forward and reverse particle transfers must be equal, i.e.,

$$
\mathscr{P}_{\alpha \beta}=\mathscr{P}_{\beta \alpha}
$$

From equation (11) we are able to determine the acceptance criterion for a reversible transfer move as

$$
\begin{aligned}
\mathscr{P}_{1}^{\mathrm{t}} & =\frac{\mathscr{P}_{\alpha \beta}^{\text {criterion }}}{\mathscr{P}_{\beta \alpha}^{\text {criterion }}} \\
& =\frac{\left(N^{a}-1\right) !\left(N^{b}-1\right) !}{N^{a} ! N^{b} !} \times \frac{\mathscr{P}^{\text {subsystem } b}}{\mathscr{P}^{\text {subsystem } a}} \times \frac{\mathscr{P}_{\beta}^{\text {particle } b}}{\mathscr{P}_{\alpha}^{\text {particle } a}} \times \frac{\mathscr{P}^{\text {position } a}}{\mathscr{P}^{\text {position } b}} \times \frac{\mathscr{P}_{\beta}^{\text {Gibbs }}}{\mathscr{P}_{\alpha}^{\text {Gibbs }}} \\
& =\exp \left[\ln \left(\frac{N^{a} V^{b}}{\left(N^{b}+1\right) V^{a}}\right)-\frac{\Delta U^{a}}{k T}-\frac{\Delta U^{b}}{k T}\right]
\end{aligned}
$$

This result is identical to the original transfer criterion (7) proposed by Panagiotopoulos for simulations in the Gibbs ensemble. This clearly means that the original algorithm and transfer criterion comply with the condition of microscopic reversibility.

We propose an alternative algorithm (algorithm 2) which satisfies the condition of microscopic reversibility and involves the random selection of the particle to be transferred from one of the $N$ particles of the total system regardless of which subsystem it is in; the particle is then transferred to the other subsystem. The means that the probability of choosing the particles is now $\mathscr{P}^{\text {particle }}=1 / N$ for all configurations. Since the choice of a particular particle for the transfer determines the donor and recipient subsystems, the probability of choosing a given subsystem is not included in overall transfer probabilities (8) and (9). As before, the sum over the $N^{a} ! N^{b}$ ! and $\left(N^{a}-1\right) !\left(N^{b}+1\right)$ ! possible permutations of the labels which we use in the actual simulation for configurations $\alpha$ and $\beta$ has to be taken into account. Following a procedure similar to that for algorithm 1, the overall acceptance criterion 
for algorithm 2 is given by

$$
\begin{aligned}
\mathscr{P}_{2}^{\mathrm{t}} & =\frac{\mathscr{P}_{\alpha \beta}^{\text {criterion }}}{\mathscr{P}_{\beta \alpha}^{\text {criterion }}} \\
& =\frac{\left(N^{a}-1\right) !\left(N^{b}+1\right) !}{N^{a} ! N^{b} !} \times \frac{\mathscr{P}^{\text {particle }}}{\mathscr{P}^{\text {particle }}} \times \frac{\mathscr{P}^{\text {position } a}}{\mathscr{P}^{\text {position } b}} \times \frac{\mathscr{P}_{\beta}^{\text {Gibbs }}}{\mathscr{P}_{\alpha}^{\text {Gibbs }}} \\
& =\frac{N^{b}+1}{N^{a}} \exp \left[\ln \left(\frac{N^{a} V^{b}}{\left(N^{b}+1\right) V^{a}}\right)-\frac{\Delta U^{a}}{k T}-\frac{\Delta U^{b}}{k T}\right] \\
& =\exp \left[\ln \left(\frac{V^{b}}{V^{a}}\right)-\frac{\Delta U^{a}}{k T}-\frac{\Delta U^{b}}{k T}\right] .
\end{aligned}
$$

It is clear that in this case we do not require a knowledge of the numbers of particles in each subsystem; this is now similar to conventional Monte Carlo simulations in the $N V T$ and $N P T$ ensembles. In the case of an ideal gas of non-interacting particles, equation (13) becomes

$$
\mathscr{P}_{2}^{\mathrm{t}}=\frac{V^{b}}{V^{a}},
$$

which makes physical sense since the probability of a particle being in a particular subsystem is proportional to the volume of that subsystem.

In table 1 we present results for the vapour-liquid equilibria of an $N=512$ square-well system with a range of $\lambda=1.5$ (see [16] for details). The chemical potentials in the coexisting phases have been calculated using the technique proposed by Smit and Frenkel [10] for simulations in the Gibbs ensemble; this is a variant of the Widom [17] particle insertion method. The results obtained using the original algorithm (algorithm 1) and transfer criterion (12) of Panagiotopoulos are seen to be indistinguishable from those obtained using our algorithm 2 with transfer criterion (13) within the error bars of the data. We have also examined smaller systems of $N=64$ particles and found that the two criteria are still essentially equivalent. This result is not unexpected since both algorithms are equivalent in the sense that the particle transfers are reversible.

Table 1. Vapour-liquid coexistence data obtained from Gibbs ensemble simulations of $N=512$ square wells with a range of $\lambda=1.5$ for algorithm 1 with criterion (12), and algorithm 2 with criterion (13). The reduced density $\rho^{*}=N \sigma^{3} / V$ and chemical potential $\mu^{*}=\mu /(k T)$ are given for the coexisting vapour $\mathrm{v}$ and liquid 1 phases as a function of

\begin{tabular}{|c|c|c|c|c|c|c|}
\hline Algorithm & Criterion & $T^{*}$ & $\rho_{v}^{*}$ & $\rho_{1}^{*}$ & $\mu_{\mathrm{v}}^{*}$ & $\mu_{1}^{*}$ \\
\hline 1 & (12) & 1.00 & $0.039 \pm 0.002$ & $0.659 \pm 0.015$ & $-3.74 \pm 0.05$ & $-3.73 \pm 0.06$ \\
\hline 2 & (13) & $1 \cdot 00$ & $0.038 \pm 0.002$ & $0.659 \pm 0.016$ & $-3.75 \pm 0.05$ & $-3.75 \pm 0.05$ \\
\hline 1 & (12) & $1 \cdot 12$ & $0.078 \pm 0.004$ & $0.579 \pm 0.023$ & $-3.29 \pm 0.07$ & $-3.29 \pm 0.07$ \\
\hline 2 & (13) & $1 \cdot 12$ & $0.085 \pm 0.004$ & $0.580 \pm 0.024$ & $-3.28 \pm 0.06$ & $-3.29 \pm 0.07$ \\
\hline 1 & (12) & $1 \cdot 20$ & $0 \cdot 151 \pm 0.020$ & $0.457 \pm 0.061$ & $-3.01 \pm 0.11$ & $-3.02 \pm 0.12$ \\
\hline 2 & (13) & $1 \cdot 20$ & $0.179 \pm 0.038$ & $0.406 \pm 0.137$ & $-3.01 \pm 0.12$ & $-3.01 \pm 0.11$ \\
\hline
\end{tabular}
the temperature $T^{*}=k T / \epsilon$. Algorithm 1 corresponds to choosing the donor subsystem at random, and then randomly choosing a particle to transfer from that subsystem. Algorithm 2 corresponds to randomly choosing a particle from the $N$ of the total system regardless of which subsystem it is in. 
It is instructive to investigate the efficiency of these two schemes. Let us consider an ideal gas for which the probability of acceptance (12) of algorithm 1 becomes

$$
\mathscr{P}_{1}^{\text {,ig }}=\frac{N^{a} V^{b}}{\left(N^{b}+1\right) V^{a}} \approx \frac{\rho^{a}}{\rho^{b}}
$$

and for algorithm 2 (13)

$$
\mathscr{P}_{2}^{\mathrm{t}, \mathrm{ig}}=\frac{V^{b}}{V^{a}} .
$$

If the two subsystems are in equilibrium, the probability of the transfers being accepted for algorithm 1 is one; for an ideal gas the two subsystems must have the same number density, i.e., $\rho^{a}=\rho^{b}$. This algorithm is therefore more efficient than algorithm 2. In fact, one would intuitively expect this since at equilibrium the number of particles added to a subsystem should be equal to the number of particles removed from that subsystem. If one uses an algorithm in which there are more attempts to insert particles than attempts to remove particles (i.e., algorithm 2), the acceptance criterion will ensure that a proportional number of attempts will be rejected.

When dealing with mixtures, there are some cases in which one would choose to use an alternative algorithm. A number of these algorithms together with the corresponding transfer criteria are presented in the following section.

\section{Gibbs ensemble Monte Carlo simulations of mixtures}

In the case of mixtures we must examine the transfers of particles of a given type to and from the two subsystems; a detailed discussion of the statistical mechanics of the isothermal-isobaric version of the Gibbs ensemble for mixtures is given elsewhere [5]. To simplify the analysis we consider a binary mixture of components 1 and 2 . In their original algorithm for mixtures, which we refer to as algorithm $1 \mathrm{~m}$, Panagiotopoulos et al. [7] suggest that the donor and recipient subsystems should be chosen at random, the type of particle should be chosen randomly with a fixed but otherwise arbitrary probability, and finally a random particle of that type in the donor subsystem should be chosen and transferred to a random position in the recipient subsystem. As for the pure component fluid of the previous section, the overall transfer probability when a particle of type 1 is transferred from subsystem $a$ to subsystem $b$ is proportional to

$$
\begin{aligned}
\mathscr{P}_{\alpha \beta}= & N_{1}^{a} ! N_{1}^{b} ! N_{2}^{a} ! N_{2}^{b} ! \times \mathscr{P}^{\text {subsystem } a} \times \mathscr{P}^{\text {type } 1} \times \mathscr{P}_{\alpha}^{\text {particle } 1, a} \times \mathscr{P}^{\text {position } b} \\
& \times \mathscr{P}_{\alpha, \mathrm{m}}^{\text {Gibbs }} \times \mathscr{P}_{\alpha \beta}^{\text {criterion }},
\end{aligned}
$$

where $N_{1}^{a} ! N_{1}^{b} ! N_{2}^{a} ! N_{2}^{b}$ ! represents the possible number of permutations of the labels for configuration $\alpha$ of the mixture, $\mathscr{P}^{\text {type }} 1$ is the probability of choosing a component of type $1, \mathscr{P}_{\alpha}^{\text {particle } 1, a}$ is the probability of choosing a given particle of type 1 in subsystem $a$,

$$
\begin{aligned}
\mathscr{P}_{a, \mathrm{~m}}^{\text {Gibbs }}= & \exp \left[\ln \left(\frac{N_{1} !}{N_{1}^{a} ! N_{1}^{b} !}\right)+\ln \left(\frac{N_{2} !}{N_{2}^{a} ! N_{2}^{b} !}\right)+N^{a} \ln V^{a}+N^{b} \ln V^{b}\right. \\
& \left.-\frac{P V^{a}}{k T}-\frac{P V^{b}}{k T}-\frac{U^{a}\left(N^{a}\right)}{k T}-\frac{N^{b}\left(N^{b}\right)}{k T}\right]
\end{aligned}
$$


is the probability that the overall system is in configuration $\alpha$ for an isothermalisobaric Gibbs ensemble simulation of the mixture [5], and the other probabilities have the same meanings as for the pure fluid. Here, $N_{i}$ and $N_{i}^{j}$ are the numbers of particles of type $i$ in the total system and in subsystem $j$; the composition of particles of type $i$ in subsystem $j$ is given by $x_{i}^{j}=N_{i}^{j} / N^{j}$. Similarly, the probability of transferring a particle of type 1 from subsystem $b$ back to subsystem $a$ is proportional to

$$
\begin{aligned}
\mathscr{P}_{\beta \alpha}= & \left(N_{1}^{a}-1\right) !\left(N_{1}^{b}+1\right) ! N_{2}^{a} ! N_{2}^{b} ! \times \mathscr{P}^{\text {subsystem } b} \times \mathscr{P}^{\text {type } 1} \times \mathscr{P}_{\beta}^{\text {particle } 1, b} \\
& \times \mathscr{P}^{\text {position } a} \times \mathscr{P}_{\beta, \mathrm{m}}^{\text {Gibbs }} \times \mathscr{P}_{\beta \alpha}^{\text {criterion }},
\end{aligned}
$$

where for configuration $\beta,\left(N_{1}^{a}-1\right) !\left(N_{1}^{b}+1\right) ! N_{2}^{a} ! N_{2}^{b}$ ! represents the possible number of permutations of the labels, $\mathscr{P}_{\beta}^{\text {particle } 1, b}$ is the probability of choosing a given particle of type 1 in subsystem $b$, and

$$
\begin{aligned}
\mathscr{P}_{\beta, \mathrm{m}}^{\text {Gibbs }}= & \exp \left[\ln \left(\frac{N_{1} !}{\left(N_{1}^{a}-1\right) !\left(N_{1}^{b}+1\right) !}\right)+\ln \left(\frac{N_{2} !}{N_{2}^{a} ! N_{2}^{b} !}\right)+\left(N^{a}-1\right) \ln V^{a}\right. \\
& \left.+\left(N^{b}+1\right) \ln V^{b}-\frac{P V^{a}}{k T}-\frac{P V^{b}}{k T}-\frac{U^{a}\left(N^{a}-1\right)}{k T}-\frac{U^{b}\left(N^{b}+1\right)}{k T}\right] .
\end{aligned}
$$

As for the pure system, the probabilities of choosing either subsystem $a$ or $b$ are equal, i.e., $\mathscr{P}_{\alpha}^{\text {subsystem } a}=\mathscr{P}_{\beta}^{\text {subsystem } b}=\frac{1}{2}$, as are the probabilities of choosing a particle of type 1 or 2 , i.e., $\mathscr{P}^{\text {type } 1}=\mathscr{P}^{\text {type } 2}=\frac{1}{2}$; these just form part of the constant of proportionality in the overall probability for the transfer. The probabilities of choosing a particle of type 1 in subsystem $a$ of configuration $\alpha$ and in subsystem $b$ of configuration $\beta$ are $\mathscr{P}_{\alpha}^{\text {particle } 1, a}=1 / N_{1}^{a}$ and $\mathscr{P}_{\beta}^{\text {particle } 1, b}=1 /\left(N_{1}^{b}+1\right)$, respectively. We can ensure that the particle transfer algorithm is reversible by equating the forward and backward probabilities. It is gratifying to see that the imposition of the condition of microscopic reversibility leads directly to the original transfer criterion of Panagiotopoulos et al. [7] which, in this case, is given by

$$
\begin{aligned}
& \mathscr{P}_{1 \mathrm{~m}}^{\mathrm{t}}= \frac{\mathscr{P}_{\alpha \beta}^{\text {criterion }}}{\mathscr{P P}_{\beta \alpha}^{\text {criterion }}} \\
&= \frac{\left(N_{1}^{a}-1\right) !\left(N_{1}^{b}+1\right) ! N_{2}^{a} ! N_{2}^{b} !}{N_{1}^{a} ! N_{1}^{b} ! N_{2}^{a} ! N_{2}^{b} !} \times \frac{\mathscr{P}^{\text {subsystem } b}}{\mathscr{P}_{\text {subsystem } a}} \times \frac{\mathscr{P}^{\text {type 1 }}}{\mathscr{P}^{\text {type } 1}} \times \frac{\mathscr{P}_{\beta}^{\text {particle } 1, b}}{\mathscr{P}_{\alpha}^{\text {particle 1,a }}} \\
& \times \frac{\mathscr{P}_{\text {position } a}^{\mathscr{P}^{\text {position } b}} \times \frac{\mathscr{P}_{\beta, \mathrm{m}}^{\text {Gibbs }}}{\mathscr{P}_{\alpha, \mathrm{m}}^{\text {Gibbs }}}}{=} \\
& \exp \left[\ln \left(\frac{N_{1}^{a} V^{b}}{\left(N_{1}^{b}+1\right) V^{a}}\right)-\frac{\Delta U^{a}}{k T}-\frac{\Delta U^{b}}{k T}\right]
\end{aligned}
$$

An identical expression can be obtained for component 2 in terms of $N_{2}^{a}$ and $N_{2}^{b}$.

Next we consider the generalization of algorithm 2 for pure fluids to mixtures (algorithm $2 \mathrm{~m}$ ). Now the particle which is to be transferred is chosen randomly from one of the $N$ particles of the total system regardless of which subsystem it is in or what species it is. Following the same procedure as before, the forward and reverse probabilities of transferring a particle of type 1 from subsystem $a$ to subsystem $b$ are still obtained from equations (17) and (19), but now $\mathscr{P}^{\text {subsystem } a}, \mathscr{P}^{\text {subsystem } b}$ and $\mathscr{P}^{\text {type } 1}$ are not included since the particles are chosen regardless of the subsystem or the 
type, and $\mathscr{P}_{\alpha}^{\text {particle } 1, a}=\mathscr{P}_{\beta}^{\text {particle } 1, b}=1 / N$. As with the original algorithm $1 \mathrm{~m}$, we can comply with the condition of microscopic reversibility by ensuring that the forward and reverse moves have the same probability. The transfer criterion for algorithm $2 \mathrm{~m}$ is given by

$$
\begin{aligned}
\mathscr{P}_{2 \mathrm{~m}}^{\mathrm{t}} & =\frac{\mathscr{P}_{\alpha,}^{\text {criterion }}}{\mathscr{P P}_{\beta \alpha}^{\text {criterion }}} \\
& =\frac{\left(N_{1}^{a}-1\right) !\left(N_{1}^{b}+1\right) ! N_{2}^{a} ! N_{2}^{b} !}{N_{1}^{a} ! N_{1}^{b} ! N_{2}^{a} ! N_{2}^{b} !} \times \frac{\mathscr{P}_{\beta}^{\text {particle } 1, b}}{\mathscr{P}_{\alpha}^{\text {particle } 1, a}} \times \frac{\mathscr{P}^{\text {position } a}}{\mathscr{P}^{\text {position } b}} \times \frac{\mathscr{P}_{\beta, \mathrm{m}}^{\text {Gibbs }}}{\mathscr{P}_{\alpha, \mathrm{m}}^{\text {Gibbs }}} \\
& =\left(\frac{N_{1}^{b}+1}{N_{1}^{a}}\right) \exp \left[\ln \left(\frac{N_{1}^{a} V^{b}}{\left(N_{1}^{b}+1\right) V^{a}}\right)-\frac{\Delta U^{a}}{k T}-\frac{\Delta U^{b}}{k T}\right] \\
& =\exp \left[\ln \left(\frac{V^{b}}{V^{a}}\right)-\frac{\Delta U^{a}}{k T}-\frac{\Delta U^{b}}{k T}\right]
\end{aligned}
$$

which is identical to criterion (13) for the corresponding algorithm 2 of pure fluids.

Before we compare the results of simulations using algorithms $1 \mathrm{~m}$ and $2 \mathrm{~m}$ for a specific system, it is interesting to examine an alternative algorithm $3 \mathrm{~m}$ which has already been used for mixtures containing square-well molecules [5]. The algorithm involves choosing the donor and recipient subsystems at random, and then randomly choosing the particle which is to be transferred from the donor subsystem regardless of its type, and transferring it to a random position in the recipient subsystem. In this case the probabilities for the forward and reverse transfers of a particle of type 1 to and from subsystems $a$ and $b$ are still proportional to equations (17) and (19), but $\mathscr{P}^{\text {type } 1}$ is not included, and $\mathscr{P}_{a}^{\text {particle } 1, a}=1 / N^{a}$ and $\mathscr{P}_{\beta}^{\text {particle } 1, b}=1 /\left(N^{b}+1\right)$. The reversible transfer criterion is now given by

$$
\begin{aligned}
\mathscr{P}_{3 \mathrm{~m}}^{\mathrm{t}}= & \frac{\mathscr{P}_{\alpha \beta}^{\text {criterion }}}{\mathscr{P P}_{\beta \alpha}^{\text {criterion }}} \\
= & \frac{\left(N_{1}^{a}-1\right) !\left(N_{1}^{b}+1\right) ! N_{2}^{a} ! N_{2}^{b} !}{N_{1}^{a} ! N_{1}^{b} ! N_{2}^{a} ! N_{2}^{b} !} \times \frac{\mathscr{P}^{\text {subsystem } b}}{\mathscr{P}^{\text {subsystem } a}} \times \frac{\mathscr{P}_{\beta}^{\text {particle } 1, b}}{\mathscr{P}_{\alpha}^{\text {particle } 1, a}} \\
& \times \frac{\mathscr{P}^{\text {position } a}}{\mathscr{P}^{\text {position } b}} \times \frac{\mathscr{P}_{\beta, \mathrm{m}}^{G \text { ibbs }}}{\mathscr{P}_{\alpha, \mathrm{m}}^{\mathrm{Gibb}}} \\
= & \left(\frac{N_{1}^{b}+1}{N_{1}^{a}}\right)\left(\frac{N^{a}}{N^{b}+1}\right) \exp \left[\ln \left(\frac{N_{1}^{a} V^{b}}{\left(N_{1}^{b}+1\right) V^{a}}\right)-\frac{\Delta U^{a}}{k T}-\frac{\Delta U^{b}}{k T}\right], \\
= & \exp \left[\ln \left(\frac{N^{a} V^{b}}{\left(N^{b}+1\right) V^{a}}\right)-\frac{\Delta U^{a}}{k T}-\frac{\Delta U^{b}}{k T}\right] .
\end{aligned}
$$

The final expression for the mixture is now the same as the corresponding expression for the pure component system (see criterion (7)).

It is important to stress that algorithms $1 \mathrm{~m}, 2 \mathrm{~m}$ and $3 \mathrm{~m}$ with the corresponding criteria (21), (22), and (23) are completely equivalent since they are all reversible. This was borne out in practice for algorithms $1 \mathrm{~m}$ and $3 \mathrm{~m}$ in the case of mixtures containing square-well molecules [5]; the calculated chemical potentials of each component were found to be equal in the two subsystems, confirming that the unique conditions for phase equilibria were satisfied. This is demonstrated again here for the 
three algorithms. The results of constant-pressure Gibbs ensemble simulations for the liquid-liquid phase equilibria of mixtures of $N=512$ symmetrical square wells with $\lambda=1.5$ in which the unlike interaction is purely repulsive are presented in table 2 (see [5] for details of the simulations). It is clear that the data corresponding to the original Panagiotopoulos et al. scheme (algorithm $1 \mathrm{~m}$ ) are virtually indistinguishable from the data corresponding to the alternative algorithms $2 \mathrm{~m}$ and $3 \mathrm{~m}$. The three algorithms describe the liquid-liquid coexistence correctly and ensure that the chemical potentials of each component in the two phases are the same.

In order to investigate the efficiency of the three algorithms, we again consider the case of the ideal gas. For a mixture of ideal gases, the acceptance criterion (21) of algorithm $1 \mathrm{~m}$ reduces to

$$
\mathscr{P}_{1 \mathrm{~m}}^{\mathrm{t}, \mathrm{ig}}=\frac{N_{1}^{a} V^{b}}{\left(N_{1}^{b}+1\right) V^{a}} \approx \frac{x_{1}^{a} \rho^{a}}{x_{1}^{b} \rho^{b}},
$$

the acceptance criterion (22) of algorithm $2 \mathrm{~m}$ to

$$
\mathscr{P}_{2 \mathrm{~m}}^{\mathrm{t}, \mathrm{ig}}=\frac{V^{b}}{V^{a}},
$$

and the acceptance criterion (23) of algorithm $3 \mathrm{~m}$ to

$$
\mathscr{P}_{3 \mathrm{~m}}^{\mathrm{t}, \mathrm{ig}}=\frac{N^{a} V^{b}}{\left(N^{b}+1\right) V^{a}} \approx \frac{\rho^{a}}{\rho^{b}} .
$$

Since the two subsystems of an ideal gas mixture at equilibrium are identical we have $\rho^{a}=\rho^{b}$ and $x_{1}^{a}=x_{1}^{b}$. As a consequence the probability that the transfer moves are accepted is one for algorithms $1 \mathrm{~m}$ and $3 \mathrm{~m}$, and these algorithms are therefore more efficient than algorithm $2 \mathrm{~m}$. For an ideal gas mixture there is no difference between algorithms $1 \mathrm{~m}$ and $3 \mathrm{~m}$ because at equilibrium $x_{1}^{a}=x_{1}^{b}$. For an interacting system, however, $x_{1}^{a}$ and $x_{1}^{b}$ will be different and one would expect differences in efficiency.

At equilibrium the number of particles of a particular type that is removed from a subsystem is equal to the number that is added to it. This suggests that an efficient algorithm (algorithm 4) would be one in which attempts are made to remove/add a particle in the two subsystems with equal probability, i.e., $\mathscr{P}_{\alpha}^{\text {subsystem } a}=$ $\mathscr{P}_{\beta}^{\text {subsystem } b}=\frac{1}{2}$, but where for each type of particle the total number of attempts is proportional to the total number of particles of that type, i.e., for particles of type 1 $\mathscr{P}^{\text {type } 1}=N_{1} / N$ and for particles of type $2 \mathscr{P}^{\text {type } 2}=N_{2} / N$. In this case we have the following acceptance criterion:

$$
\begin{aligned}
\mathscr{P}_{4 \mathrm{~m}}^{\mathrm{t}}= & \frac{\mathscr{P}_{\alpha \beta}^{\text {criterion }}}{\mathscr{P P}_{\beta \alpha}^{\text {criterion }}} \\
= & \frac{\left(N_{1}^{a}-1\right) !\left(N_{1}^{b}+1\right) ! N_{2}^{a} ! N_{2}^{b} !}{N_{1}^{a} ! N_{1}^{b} ! N_{1}^{a} ! N_{2}^{b} !} \times \frac{\mathscr{P}^{\text {subsystem } b}}{\mathscr{P}^{\text {subsystem } a}} \times \frac{\mathscr{P}^{\text {type } 1}}{\mathscr{P}^{\text {type } 1}} \times \frac{\mathscr{P}_{\beta}^{\text {particle } 1, b}}{\mathscr{P}_{\alpha}^{\text {particle } 1, a}} \\
& \times \frac{\mathscr{P}^{\text {position } a}}{\mathscr{P}^{\text {position } b}} \times \frac{\mathscr{P}_{\beta, \mathrm{m}}^{\text {Gibs }}}{\mathscr{P}_{\alpha, \mathrm{m}}^{\text {Gibs }}} \\
= & \exp \left[\ln \left(\frac{N_{1}^{a} V^{b}}{\left(N_{1}^{b}+1\right) V^{a}}\right)-\frac{\Delta U^{a}}{k T}-\frac{\Delta U^{b}}{k T}\right],
\end{aligned}
$$




\begin{tabular}{|c|c|c|}
\hline 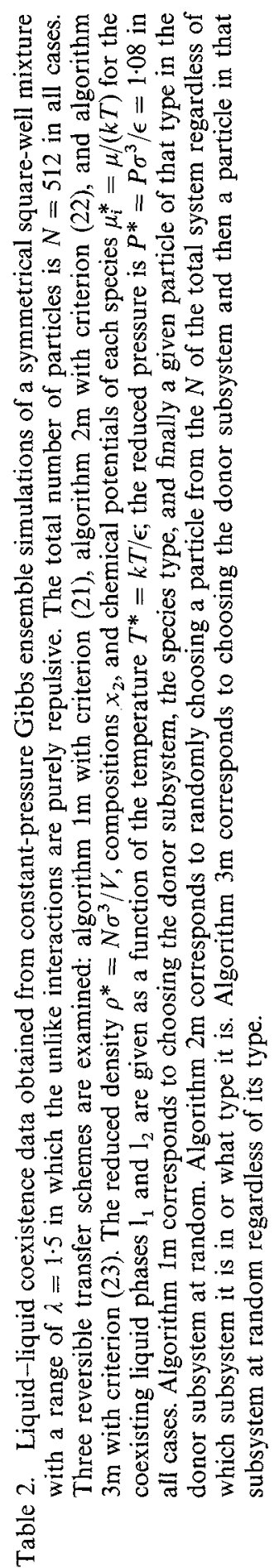 & 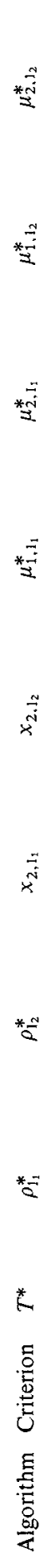 & 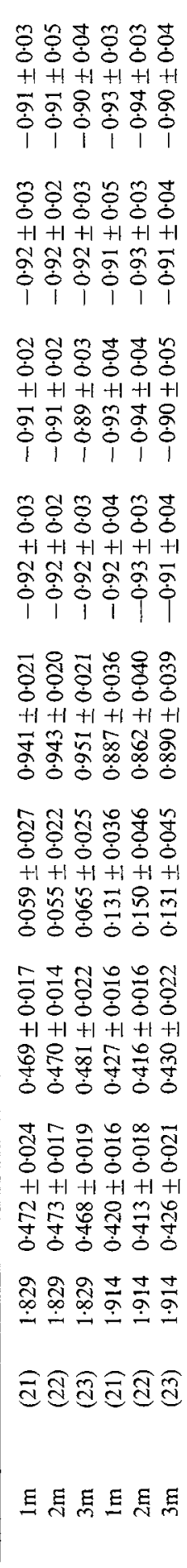 \\
\hline
\end{tabular}


which is identical to the original transfer criterion (21). The efficiency of algorithm 4 will have to be examined for a mixture other than the symmetrical one studied here since in this case we use $N_{1}=N_{2}$ so that $\mathscr{P}^{\text {type } 1}=\mathscr{P}^{\text {type } 2}=\frac{1}{2}$ as for algorithm $1 \mathrm{~m}$.

These arguments suggest that the original algorithm is the most efficient when the number of particles of the two components are equal. The other algorithms may be of more use when the number of particles in the two phases are very different.

\section{Conclusion}

In this paper we focus on the use of different algorithms and transfer criteria in Gibbs ensemble Monte Carlo simulations which conform to the conditions of microscopic reversibility. The vapour-liquid and liquid-liquid phase equilibria of pure fluids and mixtures containing square-well molecules are examined as examples. We show that the original transfer schemes of Panagiotopoulos for pure fluids and mixtures are reversible in the usual Monte Carlo sense. Alternative reversible transfer algorithms are proposed and the differences are found to be negligible. The particular choice of algorithm will depend on its efficiency for the state point under investigation.

It may have struck the reader that the analysis presented in this paper for Gibbs ensemble simulations can be applied to the creation and annihilation of particles in the commonly used grand canonical simulations [6, 14, 18-21]. If the probabilities of attempting the creaction and annihilation of the particles are equal, the usual criterion is found to be reversible, as has already been shown by Nicholson and Parsonage [21].

The authors wish to thank Richard P. Sear for useful discussions. One of us (G.J.) would like to thank the Universidad de Sevilla for its hospitality and support during his study leave as Profesor Visitante in Sevilla. The research was supported in part by grants from the British Council and from the Junta de Andalucía. We also acknowledge support from the Royal Society, the Computational Initiative of the Science and Engineering Research Council, and from the Centro Informático Cientifico de Andalucia for computer hardware on which the simulations were performed.

\section{References}

[1] Gubbins, K. E., 1989, Molec. Simulation, 2, 223.

[2] Panagiotopoulos, A. Z., 1987, Molec. Phys., 61, 813.

[3] Panaglotopoulos, A. Z., and Stapleton, M. R., 1989, Fluid Phase Equilibria, 53, 133.

[4] Panagiotopoulos, A. Z., 1992, Molec. Simulation, 9, 1.

[5] Green, D. G., JaCkson, G., DE Miguel, E., and Rull, L. F., 1994, J. chem. Phys., 101, 3190.

[6] Allen, M. P., and Tildesley, D. J., 1987, Computer Simulations of Liquids (Oxford: Clarendon Press).

[7] Panagiotopoulos, A. Z., Quirke, N., Stapleton, M. R., and Tildesley, D. J., 1988, Molec. Phys., 63, 527.

[8] JACKSON, G., 1991, Molec. Phys., 72, 1365.

[9] Smit, B., De Smedt, Ph., and Frenkel, D., 1989, Molec. Phys., 68, 931.

[10] Smit, B., and Frenkel, D., 1989, Molec. Phys., 68, 951.

[11] Wood, W. W., 1981, Physics of Simple Liquids, edited by H. N. V. Temperley, J. S. Rowlinson, and G. S. Rushbrooke (Amsterdam: North-Holland), pp. 115-230.

[12] Metropolis, N. A., Rosenbluth, A. W., Rosenbluth, M. N., Teller, A. H., and Teller, E., 1953, J. chem. Phys., 21, 1087. 
[13] Woon, W. W., 1968, J. chem. Phys., 48, 415.

[14] Norman, W. W., and Filinov, V. S., 1969, High Temp. Res., 7, 216.

[15] Hill, T. L., 1960, An Introduction to Statistical Thermodynamics (Reading, MA: Addison-Wesley).

[16] Vega, L., De Miguel, E., Rull, L. F., Jackson, G., and McLure, I. A., 1992, J. chem. Phys., 96, 2296.

[17] WIDOM, B., 1963, J. chem. Phys., 39, 2802.

[18] Adams, D. J., 1974, Molec. Phys., 28, 1241.

[19] Adams, D. J., 1975, Molec. Phys., 29, 307.

[20] Rowley, L. A., Nicholson, D., and Parsonage, N., 1975, J. comp. Phys., 17, 401.

[21] Nicholson, D., and Parsonage, N., 1982, Computer Simulation and the Statistical Mechanics of Adsorption (London: Academic Press), pp. 151-156. 